\title{
Optical spectroscopy of active galactic nuclei in SA57
}

\author{
D. Trevese ${ }^{1}$, V. Zitelli ${ }^{2}$, F. Vagnetti ${ }^{3}$, K. Boutsia ${ }^{1,4}$, and G. M. Stirpe ${ }^{2}$ \\ 1 Dipartimento di Fisica, Università di Roma "La Sapienza", P.le A. Moro 2, 00185 Roma, Italy \\ e-mail: dario.trevese@roma1.infn.it \\ 2 INAF - Osservatorio Astronomico di Bologna, via Ranzani 1, 40127 Bologna, Italy \\ 3 Dipartimento di Fisica, Universitá di Roma "Tor Vergata", via della Ricerca Scientifica 1, 00133 Roma, Italy \\ ${ }^{4}$ European Southern Observatory, Karl-Schwarzschild-Straße 2, 85748 Garching, Germany
}

Received 24 September 2007 / Accepted 17 October 2007

\section{ABSTRACT}

\begin{abstract}
Context. The cosmological evolution of X-ray-selected and optically selected active galactic nuclei (AGNs) show different behaviours interpreted in terms of two different populations. The difference is evident mainly for low luminosity AGNs (LLAGNs), many of which are lost by optical photometric surveys.

Aims. We are conducting a spectroscopical study of a composite sample of AGN candidates selected in SA57 following different searching techniques, to identify low luminosity AGNs and break down the sample into different classes of objects.

Methods. AGN candidates were obtained through optical variability and/or X-ray emission. Of special interest are the extended variable objects, which are expected to be galaxies hosting LLAGNs.

Results. Among the 26 classified objects a fair number (9) show typical AGN spectra. Ten objects show Narrow Emission Line Galaxy spectra, and in most of them (8/10) optical variability suggests the presence of LLAGNs.
\end{abstract}

Key words. galaxies: active - galaxies: nuclei - galaxies: Seyfert - X-rays: galaxies

\section{Introduction}

In recent years a growing amount of evidence suggested the existence of a link between the evolution in cosmic time of galaxy and quasar (QSO) populations. Theoretical work discusses the effect of galaxy merging on the nuclear activity, through an increment of the rate of accretion onto the massive black hole, hosted in (possibly all) galaxy nuclei (Kormendy \& Richstone 1995). Observationally, the cosmic history of active galactic nuclei (AGNs) is deduced from the analysis of optical and X-ray luminosity functions (LFs) and their redshift dependence. While optical observations imply that the maximum of the QSO/AGN number density occurs at $z_{\mathrm{M}} \gtrsim 2$ independently of their absolute luminosity (Wolf et al. 2003), X-ray surveys indicate a "cosmic downsizing" with the epoch of maximum density going from $z_{\mathrm{M}} \sim 1.5$, for bright objects $\left(L_{\mathrm{X}}(2-10 \mathrm{keV}) \sim 10^{45} \mathrm{erg} \mathrm{s}^{-1}\right)$, to $z_{\mathrm{M}} \sim 0.5$ for faint ones $\left(L_{\mathrm{X}}(2-10 \mathrm{keV}) \sim 10^{42} \mathrm{erg} \mathrm{s}^{-1}\right)$ (Ueda et al. 2003; La Franca et al. 2005). It has been suggested that this behaviour is a consequence of the existence of two distinct AGN populations: the first consisting of QSOs and brighter AGNs, born at high $z$ from frequent galaxy merging in high density regions, and related to the red part of the bimodal galaxy distribution, and the second population made of smaller and gasrich galaxies still providing material for feeding smaller black holes and energizing low luminosity AGNs at later times through galaxy interactions (Cavaliere \& Menci 2007). To evaluate sample completeness and selection effects, more detailed analysis of

$\star$ Based on observations made with the William Herschel Telescope (WHT), operated by the ING, and with the Italian Telescopio Nazionale Galileo (TNG), operated on the island of La Palma by the Fundación Galileo Galilei of the INAF (Istituto Nazionale di Astrofisica), both at the Spanish Observatorio del Roque de los Muchachos of the Instituto de Astrofisica de Canarias. the LF evolution is necessary, in particular for low luminosity, optically selected AGNs, to accurately quantify the intrinsic evolution. Brighter AGNs are detected in the optical band, mainly by their non-stellar colour, i.e. their position outside the "stellar locus" in colour space.

Fainter AGNs cannot be detected in the same way, since the observed spectral energy distribution (SED) is dominated by the host galaxy and in general is non-stellar, independently of the presence of an active nucleus. Thus, probing the cosmic downsizing in optical samples requires a different selection technique. Recently Bongiorno et al. (2007) have selected a complete, volume limited sample of 130 type 1 AGNs from the catalogue of 150000 spectra obtained by the VIMOS-VLT Deep Survey, (Le Fèvre et al. 2005). From this sample they have found the first evidence that the peak in density of lower luminosity type 1 AGNs is progressively shifted towards lower redshifts.

Another way to select AGNs is based on the detection of their variability. The method, which was proposed for the first time by van den Bergh et al. (1973), has different completeness and reliability depending on the accuracy of photometric measurements and the distribution of sampling times. It also depends on the total duration of the observing campaign, since the rms variation increases with the lag between observations (Bonoli et al. 1979; Giallongo et al. 1991; Trevese et al. 1994; Vanden Berk et al. 2004; de Vries et al. 2005). It has been applied in the past to various data-sets (e.g., Trevese et al. 1989; Cristiani et al. 1990; Véron \& Hawkins 1995). It is particularly interesting in the case of low luminosity AGNs (LLAGNs), where the image is not point-like and the colour selection fails. This search for "variable galaxies" has been tested by Bershady et al. (1998, hereinafter BTK) in the field of Selected Area 57 (SA57) where other techniques such as selection by colour and by the absence of proper motion were also applied 
(Kron \& Chiu 1981; Koo et al. 1986), and a few brighter candidates were confirmed spectroscopically. A similar procedure was applied to multi-epoch Hubble Space Telescope images by Sarajedini et al. (2003, 2006), who have also shown that some of the variability selected AGNs are not detected in X-rays. We have observed SA57 in X-rays with XMM-Newton (for $67 \mathrm{ks}$ ) and obtained a catalogue of 140 AGN candidates (Trevese et al. 2007), 98 of which are identified with optical images mainly from the KPNO survey of that field (Kron 1980; Koo 1986). Candidates for spectroscopy are either selected from optical variability or from X-ray emission. In this paper we describe the result of a first part of this spectroscopic campaign.

The paper is organized as follows. Section 2 describes spectroscopic observations and data reduction, Sect. 3 describes the results and Sect. 4 contains a discussion of the results.

We adopt the concordance cosmology, $H_{0}=$ $75 \mathrm{~km} \mathrm{~s}^{-1} \mathrm{Mpc}^{-1}, \Omega_{\mathrm{m}}=0.3, \Omega_{\Lambda}=0.7$, throughout the paper.

\section{Observations and data reduction}

Observations were carried out with the fiber-fed multi-object spectrograph AF2/WYFFOS at the $4.2 \mathrm{~m}$ William Herschel Telescope (WHT), La Palma (Canary Islands - Spain), on 28-29 April 2006. In a single, partially clear night on 1 April 2006 we also obtained the spectrum of one object with DOLORES at Telescopio Nazionale Galileo (TNG), La Palma. The 1.6 arcsec diameter of the AF2/WYFFOS fibers requires a relatively accurate positioning. For this reason we re-computed the absolute $\alpha, \delta$ in the following way. We started from the catalogue of the Kitt Peak National Observatory (KPNO) survey of SA57 (Kron 1980; Koo 1986), based on $U, B_{J}, F, N$ photometry taken almost yearly between 1974 and 1989 . We cross-correlated the object positions with position in the USNO-A2.0 catalogue. After a 2- $\sigma$ rejection, the IRAF ccmap utility provides a 4 th order coordinate transformation based on 446 objects spread over the field, with $<0.2$ arcsec rms deviation in both $\alpha$ and $\delta$, with respect to USNO-A2.0. The $2-\sigma$ rejection eliminates most of the high proper motion objects whose positions underwent significant changes between the epochs of the KPNO master catalogue (1974) and the USNO-A2.0 catalogue (1956). Proper motions in the field were published by Majewski (1992). This allowed us to select the guiding stars among low proper motion $(<1.2 \mathrm{arcsec} / \mathrm{yr})$ objects and compute their position at the epoch of observation. The result was an accurate centring of all of the selected guiding stars.

Candidates were selected from three different lists: i) variable extended candidates from BTK (details on objects fainter than 22.5 were not reported in the BTK paper); ii) point-like variable objects from Trevese et al. (1989), not yet observed spectroscopically; iii) objects from a subsample of 98 among 140 X-ray detected objects from Trevese et al. (2007) (see notes to Table 1 in the next section). Some previously observed objects were automatically included by the fiber positioning software in those cases in which some of the candidates were not observable due to the crowding of the field. Observations were carried out for two successive nights in April 2006. We adopted two different configurations, such that fainter objects had a total exposure of $12 \mathrm{~h}$, i.e. were present in both configurations and were observed for two nights, while brighter objects were observed for $6 \mathrm{~h}$ during the first or the second night. Between 15 and 20 fibers spread over the field were dedicated to the sky spectrum. We used the grating $\mathrm{R} 158 \mathrm{~B}$ providing a dispersion of $2.0 \AA / \mathrm{px}$, the detector was the 2-chip EEV mosaic, read with $2 \times 2$ binning

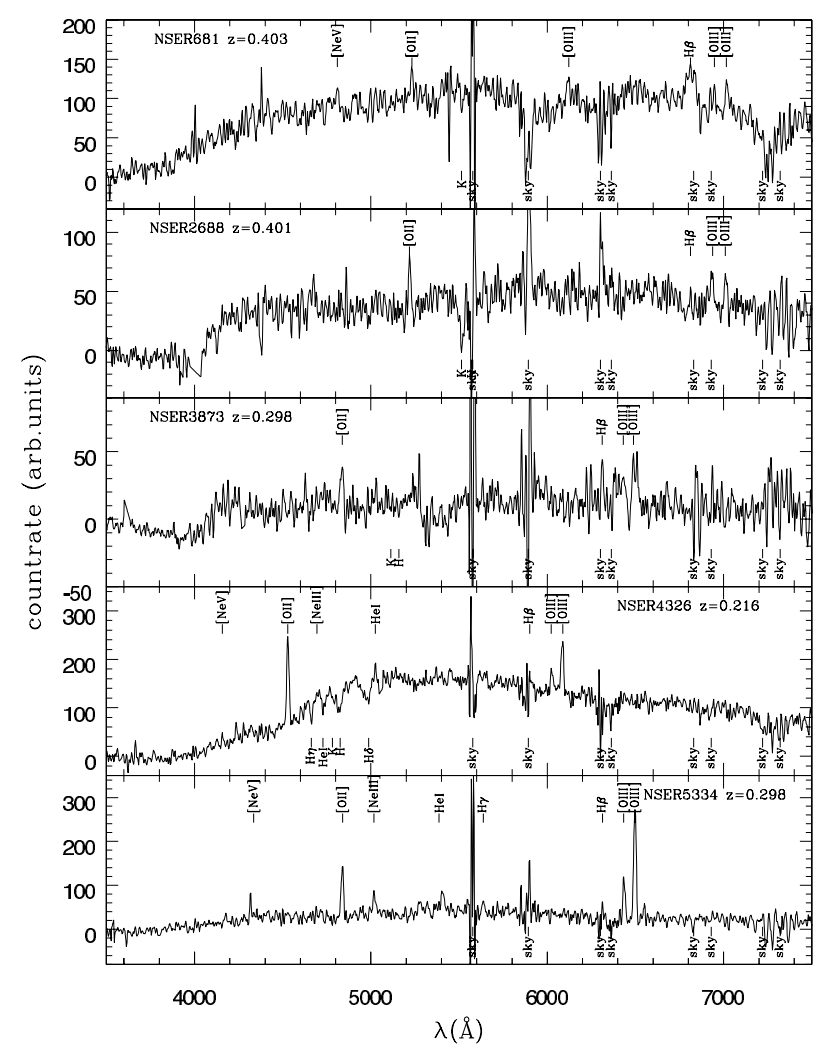

Fig. 1a. Spectra of objects observed with WYFFOS at WHT.

and the resolution was $16 \AA$. Individual observing blocks lasted $30 \mathrm{~min}$ each and were co-added to form images of $2 \mathrm{~h}$ of total exposure. For each image we also recorded arc spectra for wavelength calibration. Data reduction was carried out with standard IRAF procedures. Spectra were extracted from each (2-h) exposure and were calibrated in wavelength. An average, and high signal to noise, spectrum of the sky was obtained by combining several sky spectra. Sky subtraction was performed after a normalisation of the average sky spectrum to minimise the residuals in correspondence of the main sky emission lines. In some cases, a single sky spectrum from a fibre close to the object was subtracted instead. The spectrum of the object NSER 16338 (see Sect. 3) was also obtained with DOLORES at TNG, in long slit mode. It was obtained in a single 40 min exposure, instead of the 160 min planned, due to bad weather conditions.

\section{Results}

In the following we show the optical spectra (Figs. 1a-e and 2) and provide notes on individual objects.

\subsection{Optical spectra}

In Table 1 we list the objects. The meaning of the columns is as follows: Col. 1: serial number NSER ${ }^{1}$ in the KPNO survey (Kron 1980; Koo 1986); Cols. 2 and 3: J2000 coordinates; Cols. 4 and 5: $B_{J}$ and $F$ magnitudes; $C o l .4$ : note on variability selection; Col. 6: identification number in the X-ray catalogue of Trevese et al. (2007); Col. 7: notes on the identification; Col. 8: redshift; Col. 9: notes on redshift determination; Col. 10: classification.

\footnotetext{
1 Note that in some publications (Munn et al. 1997, BTK) the same objects are designed by a serial number equal to (NSER+100000).
} 


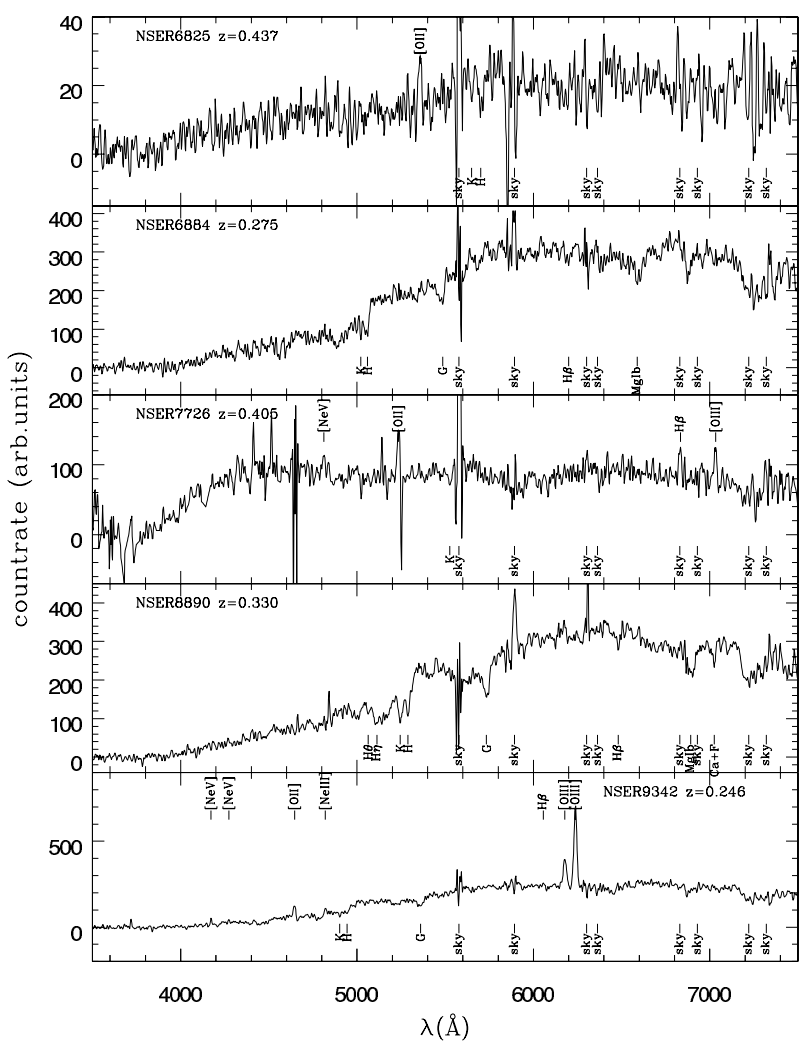

Fig. 1b. continued.

The tentative classification reported in Table 1 is based on the appearance of the optical spectra. A distinction among starburst galaxies, Seyfert 2's, and low ionization narrow emission regions (LINERs) would require the knowledge of the ([NII] $\lambda 6583) / \mathrm{H} \alpha \lambda 6563$ ) ratio which is out of our spectral range for $z \gtrsim 0.4$. Still we assume as Seyfert $2 \mathrm{~s}$ the objects

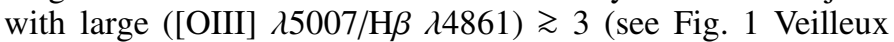
$\&$ Osterbrock 1987). These objects are classified as narrow line AGNs (NLAGNs). Where no broad lines are seen and ([OIII] $\lambda 5007 / \mathrm{H} \beta \lambda 4861$ ) is not large enough, the object may be either a starburst galaxy or a LINER and we simply designate it as narrow emission line galaxy (NELG). Objects are classified as broad line AGNs whenever the broad components are detected. It should be noted, however, that in the case of LLAGNs, this classification becomes rather "fuzzy", since the detectability of the broad lines depends on both the $\mathrm{S} / \mathrm{N}$ ratio and the relative importance of the nuclear component with respect to the host galaxy. Note that this is obviously true for any LLAGN sample, no matter how it is detected.

\subsection{Notes on individual objects}

- NSER 681: Broad-line AGN. The emission-line redshift $z_{\mathrm{e}}=$ 0.403 is confirmed as previously found by BTK. We do not see the MgII $\lambda 2798$ emission line. Instead, we reveal a broad $\mathrm{H} \beta$. Ca II K $\lambda 3934$ allows determination of an absorption-line redshift $z_{\mathrm{a}}=0.402$.

- NSER 2688: NELG. $z=0.401$ based on [OII] $\lambda 3727, \mathrm{H} \beta$, [OIII] $\lambda 4959$ and $\lambda 5007$, and on CaII K absorption.

- NSER 3873: NELG. $z=0.298$ based on [OII] $\lambda 3727, \mathrm{H} \beta$, [OIII] $\lambda 4959$ and $\lambda 5007$, and on CaII $\mathrm{H}$ and $\mathrm{K}$ absorption.

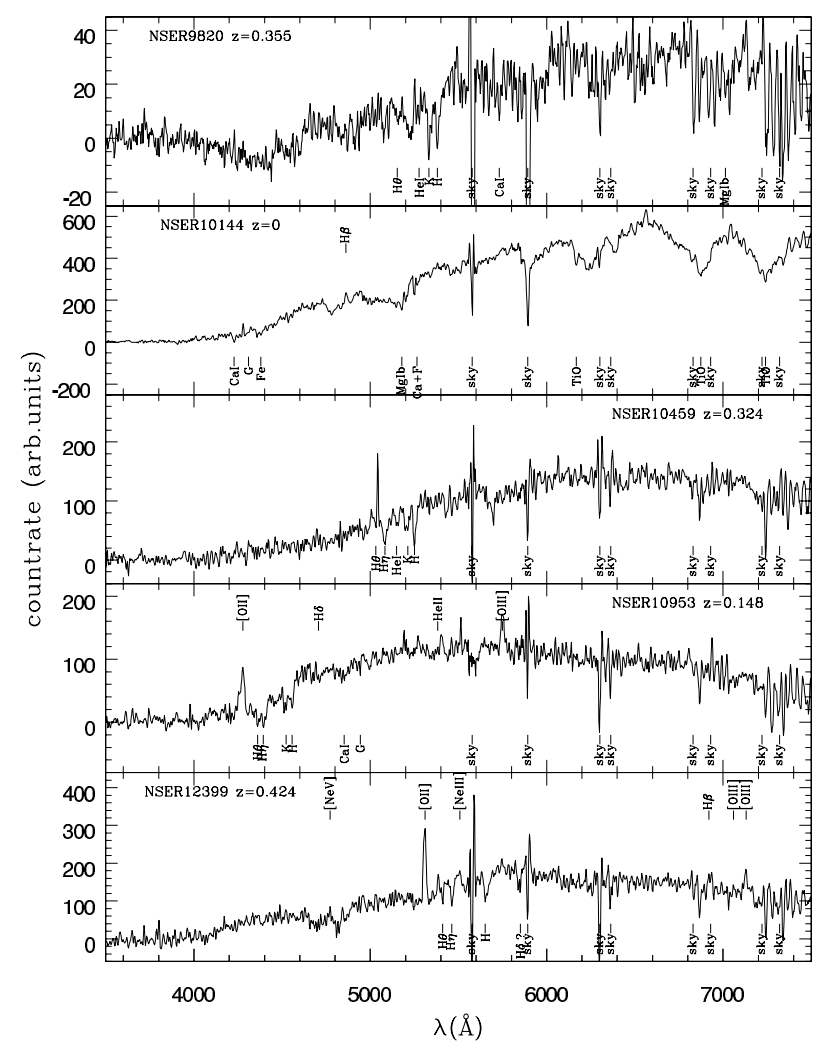

Fig. 1c. continued.

- NSER 4326: NELG with a strong [OII] 33727 and a relatively weak $[\mathrm{OIII}] \lambda 5007$. Emission and absorption features agree on a redshift $z=0.216$.

- NSER 5334: Emission-line redshift $z_{\mathrm{e}}=0.298$. The strong narrow emission lines [OII] $\lambda 3727$, [OIII] $\lambda 4959$ and $\lambda 5007$, and the low $\mathrm{H} \beta /[\mathrm{OIII}] \lambda 5007$ ratio favour a Seyfert 2 classification.

- NSER 6825: $z=0.437$ based on [OII] $\lambda 3727$ emission and on CaII $\mathrm{K}$ and $\mathrm{H}$ absorption. NELG.

- NSER 6884: No emission features. $z_{\mathrm{a}}=0.275$ based on CaII $\mathrm{K}$ and $\mathrm{H}$ and on $\mathrm{H} \beta$ and MgIb $\lambda 5175.4$. An absorptionline galaxy which can be classified XBONG due to its relatively high X-ray luminosity $\left(L_{2-10 \mathrm{keV}} \lesssim 10^{42} \mathrm{erg} / \mathrm{s}\right.$, cf. Trevese et al. 2007).

- NSER 7726: Emission features and CaII K $\lambda 3934$ absorption agree on a redshift determination $z=0.405$. Relatively strong [OII] $\lambda 3727$ and a high $\mathrm{H} \beta /[\mathrm{OIII}] \lambda 5007$ ratio suggest this object is a NELG.

- NSER 8553: We confirm the redshift $z=0.297$ by BTK. Our spectrum has worse $\mathrm{S} / \mathrm{N}$ ratio and is not reported in Fig. 1.

- NSER 8890: An absorption-line galaxy with $z_{\mathrm{a}}=0.330$ based on CaII $\mathrm{K}$ and $\mathrm{H}$ and on $\mathrm{H} \eta$ and $\mathrm{H} \theta$.

- NSER 9342: A Seyfert 2 with redshift $z=0.264$ based on [OII] $\lambda 3727$, strong [OIII] $\lambda 4959, \lambda 5007$, and on CaII H and $\mathrm{K}$ absorption.

- NSER 9820: No emission features. Absorption-line galaxy with $z_{\mathrm{a}}=0.355$ based on $\mathrm{H} \theta$, HeI $\lambda 3889$, CaII $\mathrm{K}$ and $\mathrm{H}$, $\mathrm{CaI}$ 14226.7 and MgIb $\lambda 5175.4$. Its relatively high X-ray luminosity $\left(L_{2-10 \mathrm{keV}} \lesssim 10^{42} \mathrm{erg} / \mathrm{s}\right.$, cf. Trevese et al. 2007) puts it in the range of XBONGs.

- NSER 10144: A stellar M-type spectrum with TiO molecular bands. 


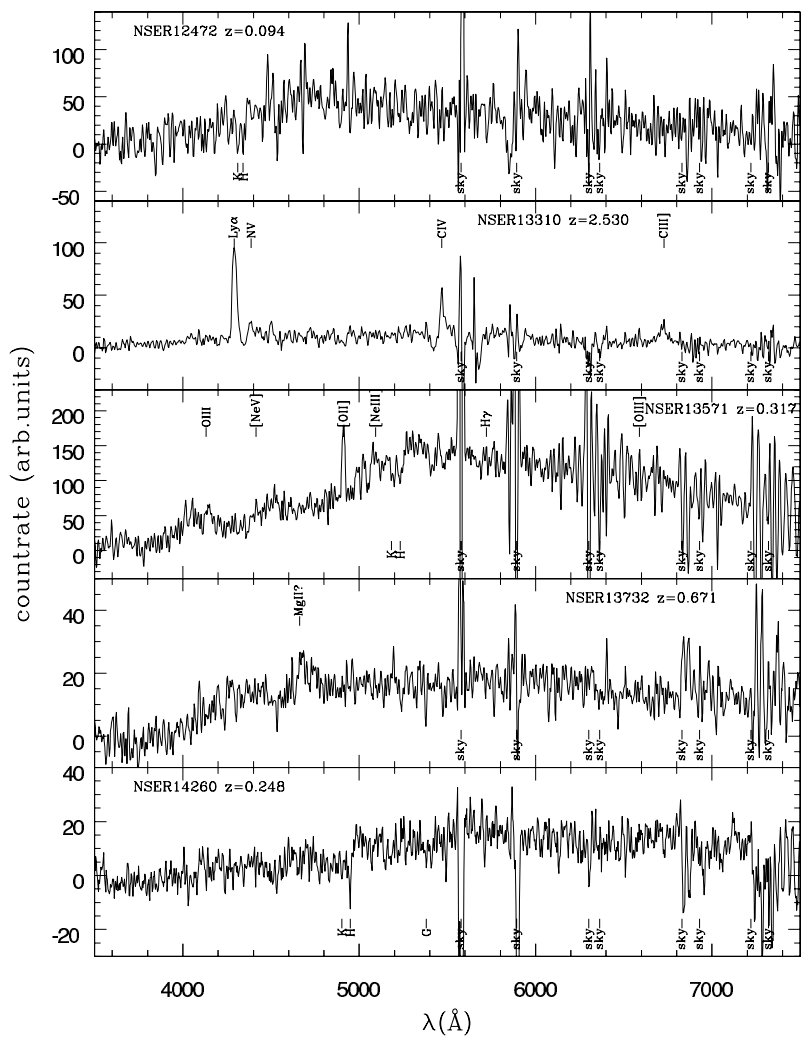

Fig. 1d. continued.

- NSER 10459: Absorption-line galaxy with $z_{\mathrm{a}}=0.324$ in agreement with the previous determination by BTK.

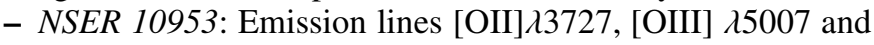
absorption features $\mathrm{CaII} \mathrm{K}$ and $\mathrm{H}, \mathrm{H} \eta$ and $\mathrm{H} \theta$ agree with a redshift $z=0.148$. NELG.

- NSER 12399: $z_{\mathrm{e}}=0.425$ based on [OII] $] 3727, \mathrm{H} \beta$ and [OIII] $\lambda 5007 . z_{\mathrm{a}}=0.424$ based on CaII H, $\mathrm{H} \eta$ and $\mathrm{H} \theta$. NELG.

- NSER 12472: No emission features. Tentative absorption redshift $z_{\mathrm{a}}=0.094$ based on CaII K and $\mathrm{H}$.

- NSER 13310: Quasar with $z_{\mathrm{e}}=2.53$ based on broad emission-lines Ly $\alpha$, NV $\lambda 1240$, CIV $\lambda 1549$ and CIII] $\lambda 1909$.

- NSER 13571: We confirm the tentative redshift determination by BTK $z=0.317$, based mainly on [OII] $\lambda 3727$ emission and CaII $\mathrm{H}$ and $\mathrm{K}$ absorption. NELG.

- NSER 13732: A single broad emission-line at $\lambda_{\mathrm{o}}=4675$, tentatively assigned to $\mathrm{MgII} \lambda 2798$, with a resulting redshift $z_{\mathrm{e}}=0.671$.

- NSER 14260: Galaxy without emission features. Tentative absorption redshift $z_{\mathrm{a}}=0.248$ based on CaII $\mathrm{K}$ and $\mathrm{H}$, and on $\mathrm{CH}-\mathrm{G}$ band $\lambda 4304.4$.

- NSER 14264: Narrow-line AGN. $z_{\mathrm{e}}=0.286$ based on [OII] $\lambda 3727,[\mathrm{OIII}] \lambda 4959$ and $\lambda 5007 . z_{\mathrm{a}}=0.287$ based on CaII K $\lambda 3934$ and H 13969 . Approximate agreement with previous determination by BTK $(z=0.287)$.

- NSER 15465: Broad-line AGN with $z_{\mathrm{e}}=0.528$ based on broad MgII $\lambda 2798$ and forbidden [OII] $\lambda 3727$.

- NSER 16338: Very weak continuum with strong narrow emission lines, [OII] $33727, \mathrm{H} \beta,[\mathrm{OIII}] \lambda 4959$ and $\lambda 5007$. A Seyfert 2 galaxy with $z_{\mathrm{e}}=0.252$. This object has been observed with both the WHT and TNG (see Figs. 1e and 2).

- NSER 16710: NELG. $z_{\mathrm{e}}=0.439$ based on [OII] $] 3727 . z_{\mathrm{a}}=$ 0.440 based on CaII $\mathrm{H}$ and $\mathrm{K}$.

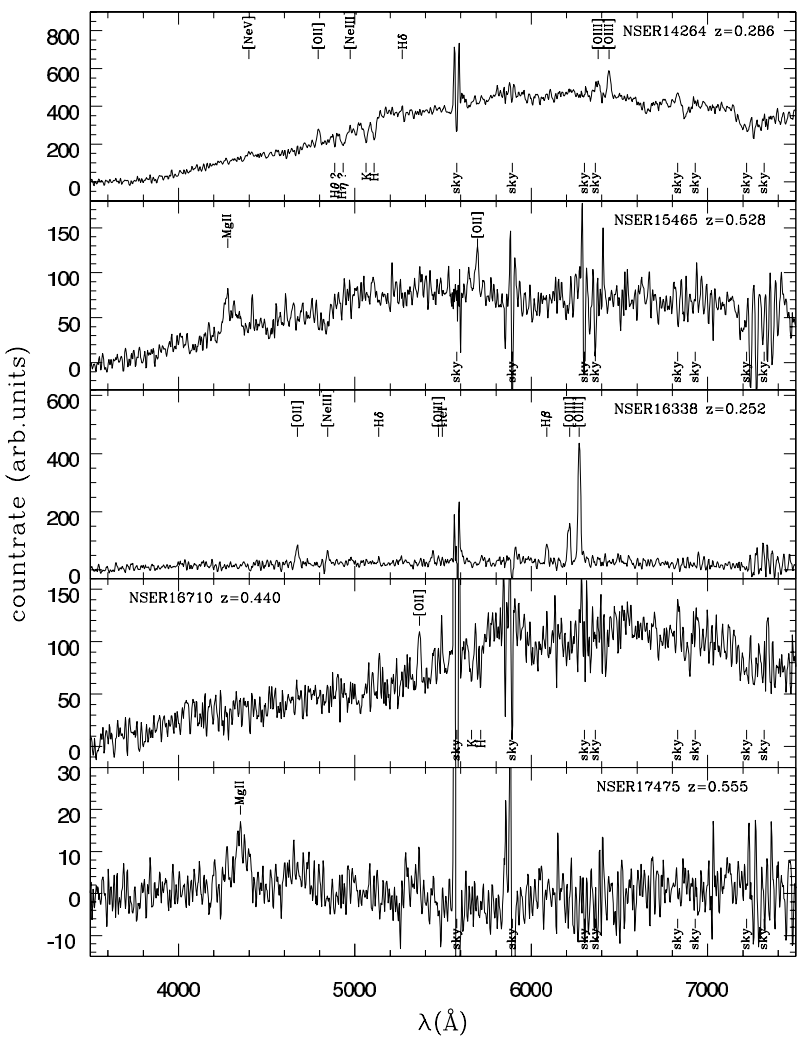

Fig. 1e. continued.

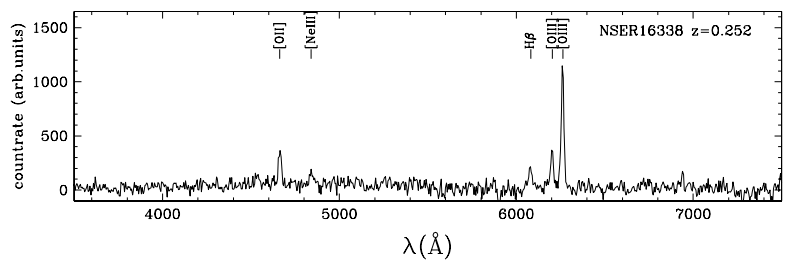

Fig. 2. Spectrum of NSER 16338 obtained with DOLORES at TNG.

- NSER 17475: Broad-line AGN, with a single feature at $\lambda_{\mathrm{o}}=$ 4352, which we identify with $\mathrm{MgII} \lambda 2798$, with a resulting redshift $z_{\mathrm{e}}=0.555$.

\section{Discussion}

Figure 3 shows the distribution in the luminosity-redshift $(L-z)$ plane of all objects with known redshift in the field of SA 57, including data from the literature and the objects of the present spectroscopic campaign (larger symbols); this contains candidates from both the X-ray catalogue of Trevese et al. (2007) and the lists of variable objects of Trevese et al. (1989, $1994)$ and BTK. The luminosity reported is computed in the $F$ band, without $K$-correction. Galaxy redshifts are taken from the KPNO redshift survey of SA 57 by Munn et al. (1997). Crowding of points around $z \sim 0.125$ corresponds to a known galaxy overdensity (Koo et al. 1984; Koo \& Kron 1987) and at $z \sim 0.24$ corresponds to the cluster Zw 1305.4+2941 analyzed in Koo et al. (1988) and Gastaldello et al. (2007). The variability-detected extended objects populate a limited region of the $L-z$ plane. This is consistent with the expectation, since the optical survey is limited to $F \lessgtr 22$. The galactic component becomes fainter and fainter at high $z$, and is swamped by the nuclear luminosity for $L_{F} \gtrsim 3 \times 10^{43} \mathrm{erg} \mathrm{s}^{-1}$, thus appearing 
Table 1. Observed objects.

\begin{tabular}{|c|c|c|c|c|c|c|c|c|c|c|}
\hline NSER & RA(2000) & $\operatorname{Dec}(2000)$ & $B_{J}$ & $F$ & $\mathrm{nv}^{a}$ & $\mathrm{SA} 57 \mathrm{X}^{b}$ & $\mathrm{ni}^{\mathrm{c}}$ & $z$ & $\mathrm{nz}^{d}$ & classification $^{e}$ \\
\hline 681 & 130805.91 & +290653.5 & 19.955 & 19.261 & EV & - & & 0.403 & $\mathrm{c}$ & BLAGN \\
\hline 2688 & 130942.82 & +291124.7 & 22.280 & 20.868 & EV & - & & 0.401 & & NELG \\
\hline 3873 & 130936.84 & +291323.9 & 22.345 & 21.503 & EV & - & & 0.298 & & NELG \\
\hline 4326 & 130853.67 & +291406.1 & 20.867 & 20.172 & EV & - & & 0.216 & & NELG \\
\hline 5334 & 130944.08 & +291547.0 & 22.235 & 21.249 & EV & - & & 0.298 & e & NLAGN \\
\hline 6825 & 130822.19 & +291807.8 & 22.920 & 21.262 & & 37 & & 0.437 & & NELG \\
\hline 6884 & 130813.52 & +291812.5 & 20.833 & 19.006 & & 38 & & 0.275 & $\mathrm{a}$ & XBONG \\
\hline 7726 & 130834.17 & +291927.0 & 21.446 & 20.409 & EV & - & & 0.405 & & NELG \\
\hline 8553 & 130713.93 & +292042.1 & 20.946 & 19.675 & EV & - & & 0.297 & $\mathrm{a}, \mathrm{c}$ & NELG \\
\hline 8890 & 130855.51 & +292110.5 & 20.964 & 19.051 & & 61 & & 0.330 & $\mathrm{a}$ & galaxy \\
\hline 9342 & 130856.78 & +292150.5 & 20.885 & 19.140 & & 66 & A & 0.246 & & NLAGN \\
\hline 9820 & 130856.74 & +292229.0 & 21.868 & 20.097 & & 71 & M & 0.355 & $\mathrm{a}$ & XBONG \\
\hline 10144 & 130900.18 & +292258.9 & 20.180 & 18.471 & & 81 & & - & & star \\
\hline 10459 & 130934.63 & +292328.1 & 21.366 & 19.682 & EV & - & & 0.324 & $\mathrm{a}, \mathrm{c}$ & galaxy \\
\hline 10953 & 130850.34 & +292415.0 & 20.960 & 20.071 & & 90 & A & 0.148 & & NELG \\
\hline 12399 & 130951.62 & +292617.9 & 21.930 & 20.710 & PV & - & & 0.424 & & NELG \\
\hline 12472 & 130729.20 & +292625.4 & 22.578 & 20.933 & & 102 & & 0.094 & $\mathrm{e}, \mathrm{u}$ & galaxy \\
\hline 13310 & 130756.71 & +292738.0 & 21.656 & 21.074 & & 109 & M & 2.530 & $\mathrm{e}$ & BLAGN \\
\hline 13571 & 130949.30 & +292800.7 & 21.713 & 20.677 & EV & - & & 0.317 & & NELG \\
\hline 13732 & 130824.04 & +292819.2 & 22.763 & 22.640 & & 115 & & 0.671 & $\mathrm{e}, \mathrm{u}$ & BLAGN \\
\hline 14260 & 130758.37 & +292908.2 & 22.223 & 20.718 & EV & - & & 0.248 & $\mathrm{a}, \mathrm{u}$ & galaxy \\
\hline 14264 & 130803.40 & +292908.8 & 20.391 & 18.782 & EV & 120 & & 0.286 & & NLAGN \\
\hline 15465 & 130917.09 & +293104.3 & 21.886 & 21.151 & PV & 127 & & 0.528 & $\mathrm{e}$ & BLAGN \\
\hline 16338 & 130730.34 & +293222.5 & 22.719 & 21.957 & EV & - & & 0.252 & $\mathrm{e}$ & NLAGN \\
\hline 16710 & 130903.87 & +293306.3 & 21.690 & 20.119 & EV & - & & 0.440 & $\mathrm{u}$ & NELG \\
\hline 17475 & 130753.14 & +293417.0 & 22.551 & 22.410 & PV & - & & 0.555 & $\mathrm{e}, \mathrm{u}$ & BLAGN \\
\hline
\end{tabular}

${ }^{a}$ Notes on variability selection. EV: extended variable; PV: pointlike variable.

${ }^{b}$ X-ray catalogue number following Trevese et al. (2007).

${ }^{c}$ Notes on optical identification. M: marginal; A: ambiguous.

${ }^{d}$ Notes on redshift. e: only emission; a: only absorption; c: confirmed redshift (see BTK); u: uncertain.

${ }^{e}$ BLAGN: broad line AGN; NLAGN: narrow line AGN; NELG: narrow emission line galaxy; XBONG: X-ray bright optically normal galaxy; galaxy: galactic spectrum with only absorption lines.

as point-like. It should be noted, however, that this does not reduce the interest of variability detection because: i) the main aim of the present survey is to detect faint AGNs at low redshift to verify the possible dependence of the evolution on intrinsic luminosity; ii) variability can detect also AGNs at higher redshift, though they appear as point-like and thus are also detectable by colour techniques. X-ray candidates span a wide range of redshift and luminosity $\left(\Delta \log L_{F} \sim 4\right)$, from relatively faint X-ray sources like starburst galaxies, to bright QSOs. Despite the spectroscopic campaign being still incomplete, so that a discussion of the LF evolution at low luminosity is still unfeasible, we find some new interesting objects indicating that the low luminosity part of our sample of AGN candidates consists of a mix of different object types. In fact, while at higher luminosities $\left(L_{F} \gtrsim 10^{43} \mathrm{erg} \mathrm{s}^{-1}\right)$ most objects are broad line AGNs, at lower luminosities most objects are either narrow line AGNs or NELGs.

Figure 4 shows the optical $F$ band versus the X-ray luminosity for the objects of the present spectroscopic campaign plus X-ray detected objects by Trevese et al. (2007) with previously known redshift. The 3- $\sigma$ upper limits in the X-ray luminosity correspond to objects not detected in the $2-10 \mathrm{keV}$ band which may be either selected through optical variability and not detected in X-rays, or detected in X-rays but in a different band.

Most of the objects whose redshift has been determined in the present work have a relatively low X-ray $(2-10 \mathrm{keV})$ luminosity. Moreover, most of the objects selected through variability are not detected in X-rays, i.e. they are likely to have a low value of the $\mathrm{X} / \mathrm{O}$ ratio, defined as the ratio of the $\mathrm{X}$-ray flux
$f_{\mathrm{X}}(2-10 \mathrm{keV})$ and the $F$-band flux $f_{F}$. They could be faint AGNs with typical nuclear X/O $(\log X / O \sim 1)$, but with the host galaxy contributing to the observed optical luminosity. Other objects, detected in X-rays, show optical spectra without emission lines, consistent with normal galaxies and luminosities $L_{\mathrm{X}}(2-10 \mathrm{keV}) \sim 10^{42} \mathrm{erg} \mathrm{s}^{-1}$. Thus they can be classified as X-ray bright optically normal galaxies (XBONGs) described by Fiore et al. (2000) and Comastri et al. (2002a,b). Different scenarios have been proposed to interpret these objects: i) selection effects hampering line detection (Hornschemeier et al. 2005): ii) heavy nuclear absorption (Comastri et al. 2002a); iii) heavy extra-nuclear absorption by the host galaxy dust (Rigby et al. 2006); iv) strong dilution by the host galaxy light (Georgantopulos \& Georgakakis 2005); v) radiatively inefficient accretion flow in low luminosity active nuclei (Yuan \& Narayan 2004). However all the above interpretations are based on the presence of an active nucleus. In particular Yuan \& Narayan (2004) postulate the existence of a transition radius in the accretion disk, below which a radiatively inefficient accretion flow (RIAF) occurs. According to Ho (1999) a RIAF can also explain the absence of a big blue bump in the spectra of LINERs (see however Maoz 2007). This suggests a relation between the two classes of objects in spite of the differences in their optical and $\mathrm{X}$-ray properties. The completion of the spectroscopic followup of our candidates will provide further data to investigate this issue.

Variability detected narrow emission line objects deserve further discussion. Let us consider first the objects we classified as Seyfert 2 s on the basis of their high [OIII]/H $\beta$ ratio. According 


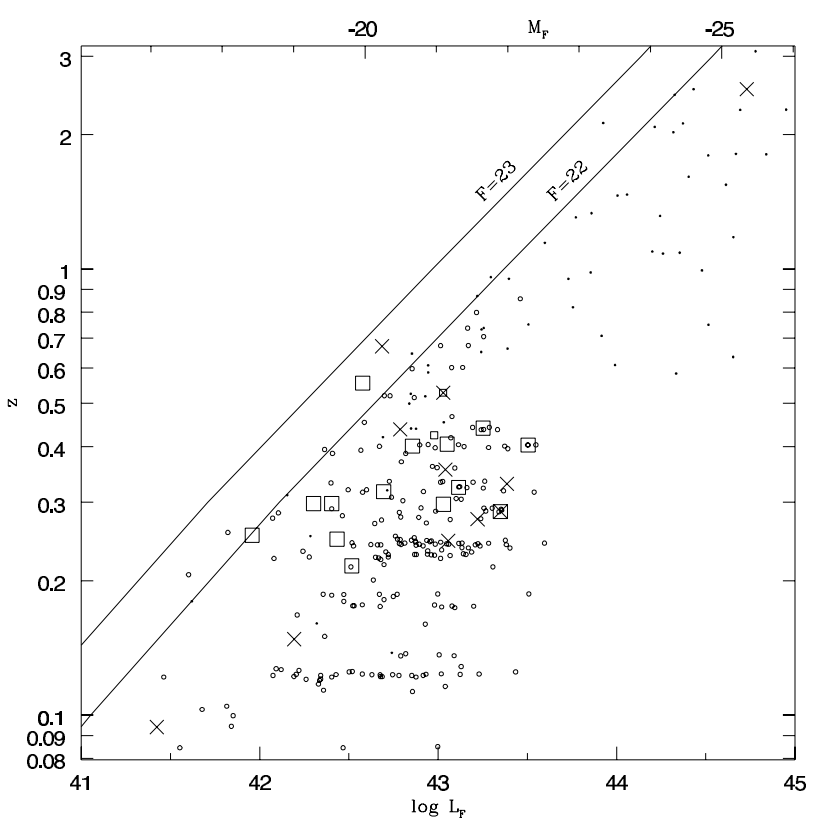

Fig. 3. Objects of SA 57 in the $L_{F}-z$ plane. From the present survey: large squares (extended variable candidates), small squares (point-like variable candidates), crosses (X-ray selected candidates). Objects with known redshift from the KPNO survey (Munn et al. 1997) are shown as: small empty circles (extended objects), small dots (point-like objects). Lines of constant magnitudes $F=22$ and $F=23$ are also shown.

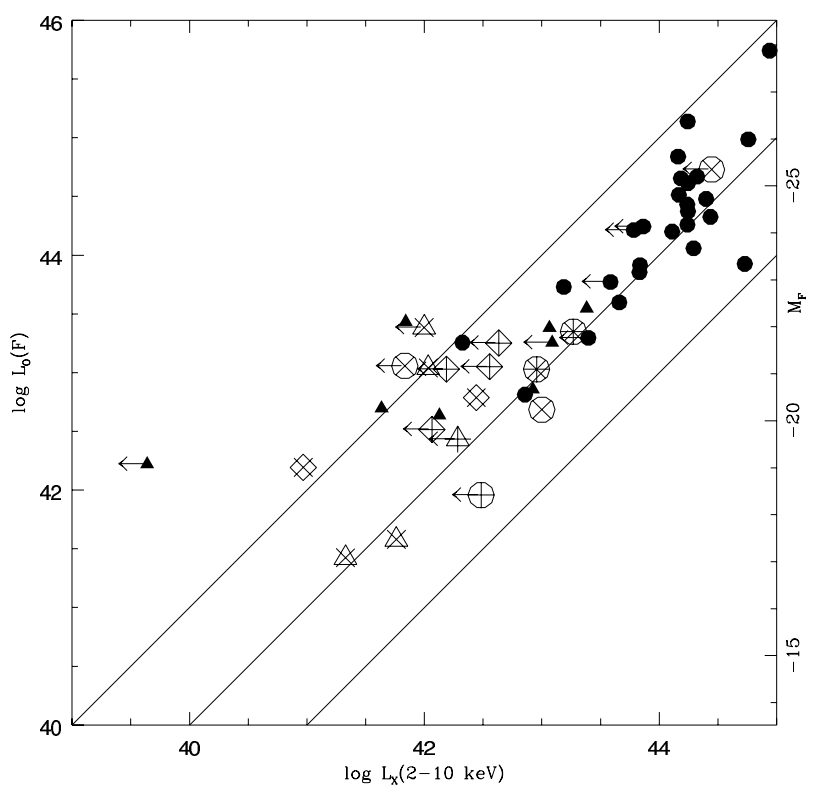

Fig. 4. $L_{F}$ vs. $L_{X}(2-10 \mathrm{keV})$. Objects with new redshifts from the present work are plotted as open symbols with a superposed " $\times$ " (X-ray selected) and/or a "+" (variability selected). According to the classification in Table 1, they are represented as: circles (type 1 and 2 AGNs), diamonds (NELGs: starbursts or LLAGNs), triangles (galactic spectra with only absorption features). Objects with previously known redshifts are plotted with filled symbols (as in Trevese et al. 2007): circles (type 1 and 2 AGNs); triangles (galaxies with redshift from Munn et al. 1997).

to the classic unified model (Antonucci \& Miller 1985) the nuclear component should be hidden by the absorbing torus. At the same time the size of the narrow line region is such that line variability should be strongly reduced. Thus, in our case, the origin of variability is unclear. Notice that variability in some type 2 objects has been observed by Klesman \& Sarajedini (2007). A possible explanation might be that the broad line region is not obscured, but intrinsically lacking, so that the variable continuum can be seen (Ghosh et al. 2007). Moreover some objects exhibit extreme spectral variations such that they appear to be of different type depending on the observing epoch (see for instance Czerny 2004, and refs. therein) as it was already noted by BTK in the case of NSER 4326 (104326 in BTK). An object of this type could be NSER 16338, which was selected on the basis of its variability during the photometric campaign in the years 1974-1989, when its magnitude was $B \sim 22.7$ and, on the basis of the present observations (April 2006), shows a very low optical continuum with only strong emission lines. Its spectrum is shown in both Figs. 1e and 2, as observed with WHT and TNG respectively. It would be interesting to monitor this object to detect possible long time scale spectral variations.

Some of the objects in Table 1 are generically classified as NELGs since we do not observe $[\mathrm{NII}] / \mathrm{H} \alpha$, while $[\mathrm{OIII}] / \mathrm{H} \beta$ is not large enough to indicate the AGN character. These objects could be either starburst galaxies, or LINERs or transition objects (TOs). However, we can look at their variability. Among the 10 NELGs in Table 1, only 2 were below the variability selection threshold and were selected solely on the basis of their X-ray emission. The other 8 NELGs were selected through their variability, and this is evidence in favour of their AGN character. In fact Maoz et al. (2005), on the basis of Hubble Space Telescope monitoring of a sample of LINERs, conclude that UV variability is detected in most, if not all, objects of this type. Moreover they conclude that this murmur of a sleeping black hole cannot be entirely explained by the variability of luminous stars but implies the presence of a non-stellar component. According to Cid-Fernandes et al. (2004) and Flohic et al. (2006), even if the AGN is present in LINERs, often its luminosity is insufficient to explain the observed emission-line intensity. In any case our results on variability selected objects favour the presence of low luminosity AGNs in at least some narrow emission line galaxies.

In summary:

- We have obtained the spectra of a composite sample of variability-selected and X-ray-selected AGN candidates.

- Some candidates show typical AGN spectra.

- One variability selected object has strong emission lines but undetected continuum, suggesting extreme spectral variation.

- 2 objects are classified as XBONGs.

- Most of the other objects are classified as NELGs, i.e. starbursts or LINERs.

- Most NELGs were variability selected suggesting that they are LINERs hosting a LLAGN.

We are continuing our spectroscopic campaign in SA57 with various telescopes, and we plan to obtain spectra of a larger sample of faint AGN candidates selected both through variability and X-ray emission. This will allow us to evaluate the relevance of the different selection effects in the study of the evolution of the faint end of the AGN luminosity function. A collection of a larger sample of variability-selected LINERs will provide a better understanding of the role of the hosted AGNs.

Acknowledgements. We thank A. Cavaliere for useful comments. We acknowledge partial support of Agenzia Spaziale Italiana and Istituto Nazionale di Astrofisica by the grant ASI/INAF No. I/023/05/0. 


\section{References}

Antonucci, R., \& Miller, J. S. 1985, ApJ, 297, 621

Bershady, M. A., Trevese, D., \& Kron, R. G. 1998, ApJ, 496, 103 (BTK)

Bongiorno, A., Zamorani, G., Gavignaud, I., et al. 2007, A\&A, 472, 443

Bonoli, F., Braccesi, A., Federici, L., Zitelli, V., \& Formiggini, L. 1979, A\&AS, 35,391

Cavaliere, A., \& Menci, N. 2007, ApJ, 664, 47

Cid-Fernandes, R., González Delgado, R. M., Schmitt, H., et al. 2004, ApJ, 605, 105

Comastri, A., Mignoli, M., Ciliegi, P., et al. 2002, ApJ, 571, 771

Comastri, A., Brusa, M., Ciliegi, P., et al. 2002, in New Visions of the X-ray Universe in the XMM-Newton and Chandra Era, ed. F. Jansen, ESA SP-488

Cristiani, S., Vio, R., \& Andreani, P. 1990, AJ, 100, 56

Czerny, B. 2004, ASP Conf. Ser., 360, 265

de Vries, W. H., Becker, R. H., White, R. L., \& Loomis, C. 2005, AJ, 129, 615

Fiore, F., La Franca, F., Vignali, C., et al. 2000, NewA, 5, 143

Flohic, H. M. L. G., Eracleous, M., Chartas, G., Shields, J. C., \& Moran, E. C. 2006, ApJ, 647, 140

Gastaldello, F., Trevese, D., Vagnetti, F., \& Fusco-Femiano, R. 2007, ApJ, in press [arXiv:0709.3168]

Geha, M., Alcock, C., Allsman, R. A., et al. 2003, ApJ, 125, 1

Georgantopulos, I., \& Georgakakis, A. 2005, MNRAS, 358, 131

Ghosh, H., Pogge, R. W., Mathur, S., Martini, P., \& Shields, J. C. 2007, ApJ, 656,105

Giallongo, E., Trevese, D., \& Vagnetti, F. 1991, ApJ, 377, 345

Ho, L. C. 1999, ApJ, 516, 672

Hornschemeier, A. E., Heckman, T. M., Ptak, A. F., Tremonti, C. A., \& Colbert, E. J. M. 2005, ApJ, 129, 86

Klesman, A., \& Sarajedini, V. 2007, ApJ, 665, 225

Koo, D. C. 1986, ApJ, 311, 651
Koo, D. C., \& Kron, R. G. 1987, IAU Symp., 124, 383

Koo, D. C., Kron, R. G., Nanni, D., Trevese, D., \& Vignato, A. 1984, in Clusters and Groups of Galaxies, ed. F. Mardirossian et al. (Dordrecht: Reidel), 159

Koo, D. C., Kron, R. G., \& Cudworth, K. M. 1986, PASP, 98, 285

Koo, D. C., Kron, R. G., Nanni, D., Trevese, D., \& Vignato, A. 1988, ApJ, 333, 586

Kormendy, J., \& Richstone, D. 1995, ARA\&A, 33, 581

Kron, R. G. 1980, ApJS, 43, 305

Kron, R. G., \& Chiu, L. G. 1981, PASP, 93, 397

La Franca, F., Fiore, F., Comastri, A., et al. 2005, ApJ, 635, 864

Le Fèvre, O., Vettolani, G., Garilli, B., et al. 2005, A\&A, 439, 845

Majewski, S. R. 1992, ApJS, 78, 87

Maoz, D. 2007, MNRAS, 377, 1696

Maoz, D., Nagar, N. M., Falcke, H., \& Wilson, A. S. 2005, ApJ, 625, 699

Munn, J. A., Koo, D. C., Kron, R. G., et al. 1997, ApJS, 109, 45

Rigby, J. R., Rieke, G. H., Donley, J. L., Alonso-Herrero, A., \& Perez-Gonzalez, P. G. 2006, ApJ, 645, 115

Sarajedini, V. L., Gilliland, R. L., \& Kasm, C. 2003, ApJ, 599, 173

Sarajedini, V. L., Koo, D. C., Phillips, A. C., et al. 2006, ApJS, 166, 69

Trevese, D., Pittella, G., Kron, R. G., Koo, D. C., \& Bershady, M. A. 1989, AJ, 98, 108

Trevese, D., Kron, R. G., Majewski, S. R., Bershady, M. A., \& Koo, D. C. 1994, ApJ, 433, 494

Trevese, D., Vagnetti, F., Puccetti, S., et al. 2007, A\&A, 469, 1211

Ueda, Y., Akiyama, M., Ohta, K., \& Miyaji, T. 2003, ApJ, 598, 886

Vanden Berk, D. E., Wilhite, B. C., Kron, R. G., et al. 2004, ApJ, 601, 692

van den Bergh, S., Herbst, E., \& Pritchet, C. 1973, AJ, 78, 375

Veilleux, \& Osterbrock 1987, ApJS, 63, 295

Véron, P., \& Hawkins, M. R. S. 1995, A\&A, 296, 665

Wolf, C., Wisotzki, L., Borch, A., et al. 2003, A\&A, 408, 499

Yuan, F., \& Narayan, R. 2004, ApJ, 612, 724 